\title{
Joint Subcarrier and Power Allocation in Channel-Aware Queue-Aware Scheduling for Multiuser OFDM
}

\author{
Chandrashekar Mohanram and Srikrishna Bhashyam
}

\begin{abstract}
In an Orthogonal Frequency Division Multiplexing (OFDM) downlink scenario, we propose joint subcarrier and power allocation for Channel-Aware Queue-Aware scheduling while allowing multiple users to share a single OFDM symbol. Our approach is to combine subcarrier and power allocation by optimizing a user's power allocation immediately after the user has been allocated a subcarrier. Simulation results show that joint subcarrier and power allocation yields a significant performance improvement compared to other existing schemes which perform subcarrier allocation with a fixed (uniform) power allocation assumption. Joint subcarrier and power allocation is also extended to band-wise allocation of subcarriers in order to help reduce signaling overhead in time varying channels. We examine the trade-off between increasing the sub-band size and the corresponding degradation in system performance for different values of the channel multipath delay spread.
\end{abstract}

Index Terms-Bit allocation, MLWDF, OFDM, OFDMA, power allocation, subcarrier allocation.

\section{INTRODUCTION}

$\mathbf{F}$ OURTH generation mobile communication systems based on Orthogonal Frequency Division Multiplexing (OFDM) will support packet data services in addition to traditional voice services. In a packet-based environment with bursty arrival of packets, the base station maintains queues to allow buffering of data for each user in the system. In case of real-time traffic, bit outage probability (buffer overflow) and delay are the two main Quality of Service (QoS) parameters to measure system performance. Efficient buffer management, essential to ensure good system performance, can be achieved when scheduling algorithms take into account the users' queue backlogs in addition to the channel conditions. Such algorithms have been proposed in [1]-[3] and belong to the Channel-Aware Queue-Aware (CAQA) class of scheduling algorithms. In [1]-[3], it has been shown that CAQA scheduling performs significantly better than Channel-Aware-Only (CAO) scheduling [4]-[10] where scheduling decisions are based on channel information alone.

In [1], the Modified Largest Weighted Delay First (MLWDF) rule is proposed for time slot allocation in Code Division Multiple Access-High Data Rate (CDMA-HDR) systems.

Manuscript received March 23, 2006; revised August 19, 2006 and October 23, 2006; accepted November 6, 2006. The associate editor coordinating the review of this letter and approving it for publication was S. Aissa. This work was performed at the Department of Electrical Engineering, Indian Institute of Technology Madras.

C. Mohanram is with Larsen and Toubro Infotech Limited, Plot Number 2530, EPIP 1st Phase, KIABD Industrial Area, Whitefield Bangalore Karnataka, 560066 India (e-mail: chandrashekar.mohanram@Intinfotech.com).

S. Bhashyam is with the Department of Electrical Engineering, Indian Institute of Technology Madras, ESB 337A, Chennai, Tamil Nadu, 600036 India (e-mail: skrishna@ee.iitm.ac.in).

Digital Object Identifier 10.1109/TWC.2007.06030103
This rule is shown to be throughput optimal ${ }^{1}$ under the assumption that only one user is scheduled in each time slot. The MLWDF rule can be applied to OFDM systems when only one user is allocated the complete OFDM symbol in any given time slot. However, due to the frequency selectivity of the channel and multiuser channel diversity, it is more efficient for multiple users to share each OFDM symbol [4]. In [2], the MLWDF rule is used at the subcarrier level instead of the OFDM symbol level to obtain improved throughput and larger admissible traffic. The MLWDF rule uses instantaneous Head-Of-Line (HOL) packet delays ${ }^{2}$ in addition to channel conditions for resource allocation. A similar rule that uses average waiting times instead of instantaneous HOL delays is proposed in [3]. However, [2], [3] optimize subcarrier allocation for a fixed power allocation, i.e., the power allocation is assumed to be given (usually uniform power allocation across all subcarriers) while subcarrier allocation is performed.

In this paper, we show that joint subcarrier and power allocation (JSPA) can provide significant gains compared to the subcarrier allocation algorithms in [2], [3]. Since optimal joint subcarrier and power allocation is very complex, we propose a sub-optimal joint subcarrier and power allocation algorithm for CAQA scheduling. The proposed algorithm (a) allows larger arrival traffic, and (b) achieves lower delay while maintaining stable queues, compared to existing algorithms which perform subcarrier allocation with a fixed power allocation assumption. An improved version of the CAQA algorithms with fixed power allocation [2], [3] is also proposed and used in the comparisons. Finally, we extend the JSPA approach to band-wise allocation of subcarriers [14] to help reduce signaling and feedback overhead. We examine the trade-off between increasing the sub-band size and the corresponding degradation in system performance for different values of the channel multipath root mean-squared (rms) delay spread $^{3}$.

JSPA is achieved by optimizing a user's power allocation immediately after the user has been allocated a subcarrier. The JSPA approach has been previously considered for CAO scheduling in [8]. In this paper, the JSPA approach is extended for CAQA scheduling. In CAO scheduling, users are assumed to have an infinite amount of data to send, i.e., all capacity available to a user would translate to throughput for the user. In a packet based environment with bursty arrival of packets,

\footnotetext{
${ }^{1} \mathrm{~A}$ scheduling rule is throughput optimal if it satisfies the property that it renders the queues at the base-station stable if any other rule can do so, i.e., it has the largest stable admission region [12].

${ }^{2} \mathrm{HOL}$ packet delay refers to the delay of the packet at the head of the queue [12].

${ }^{3}$ The rms delay spread is equal to the standard deviation of the delay of the paths weighted by the power of each path.
} 
this assumption is not true. In this paper, actual throughput achieved by each of the scheduling schemes is compared instead of a comparison of the available capacity. Furthermore, practical constraints on transmission rates are also imposed by restricting the rates to take values from a discrete set using integer bit M-ary Quadrature Amplitude Modulation (MQAM) constellations. Practical power allocation algorithms such as [13] are used instead of ideal water-filling.

In [11], joint power and bandwidth allocation has been studied in a general setting that could be applied to CDMA as well as OFDM systems. However, the results in [11] are only valid for frequency non-selective fading channels in the case of OFDM. Under this assumption, the resource allocation problem is significantly simpler and different. In this paper, the proposed JSPA approach for CAQA scheduling is designed for frequency selective channels.

\section{System Model and Problem Statement}

We assume that the base station has knowledge of the channels of all users in the system. Since channel conditions vary over a period of time, this channel state information (CSI) is updated periodically with the help of feedback channels. In this paper, we assume that the channel is constant over an entire slot (i.e., an OFDM symbol duration).

We consider a system with $M$ users, $K$ subcarriers and total bandwidth $B$. Each of the $M$ users has a queue which is implemented as a finite length first-in first-out (FIFO) buffer. The packet arrival for user $m$ is modeled as a Bernoulli process with mean arrival rate $\lambda_{m}$ (bits/time slot) i.e. a fixed size packet is admitted into user $m$ 's queue with probability $p_{m}$ at the beginning of each time slot. Let $q_{m, 0}(n)$ be the number of bits in a packet admitted into user $m$ 's queue during time slot $n$. Since finite length queues are used, a packet in the queue will be dropped if it is not scheduled for transmission within a finite duration. If $D$ is the buffer length (in packets), then the queue cannot hold any untransmitted bits from a packet arrival $D$ time slots earlier than the current time slot. Let $q_{m, d}(n)$ be the number of untransmitted bits in user $m$ 's queue from a packet arrival in time slot $(n-d)$. The number of bits in user $m$ 's queue is given by $Q_{m}(n)=\sum_{d=0}^{D-1} q_{m, d}(n)$. The HOL packet delay of user $m$ during the time slot $n$ is

$W_{m}(n)=\left\{\begin{array}{l}\arg \max _{1 \leq W \leq D}\left\{q_{m, W-1}(n)>0\right\} \text { if } Q_{m}(n)>0 \\ 0 \text { if } Q_{m}(n)=0\end{array}\right.$

In practice, packets are not expected to be served in the same time slot as they arrive. Therefore, the packet delay is set to 1 as soon as a packet arrives. Let $R_{m}(n)$ be the maximum number of bits that user $m$ can transmit during time slot $n$. For simplicity, in the remainder of this paper, $R_{m}(n)$ is just referred to as the rate for user $m$ during time slot $n$. The resultant throughput for user $m$ during time slot $n$ is $\min \left(Q_{m}(n), R_{m}(n)\right)$. Therefore, we have $Q_{m}(n+1)=$ $Q_{m}(n)-\min \left(Q_{m}(n), R_{m}(n)\right)+q_{m, 0}(n+1)$. In this paper, we maximize the long term system throughput by adaptively allocating subcarriers and power to users under a total power constraint and a bit error rate (BER) constraint i.e.,

$$
\begin{aligned}
& \max _{\mathcal{K}_{m}, P_{m, k}} \sum_{m=1}^{M} \eta_{m}, \\
\text { subject to : } & \bigcup_{m=1}^{M} \mathcal{K}_{m} \subseteq \mathcal{K}, \\
\text { where } & \mathcal{K}_{1}, \mathcal{K}_{2}, \mathcal{K}_{3}, \cdots, \mathcal{K}_{M} \text { are all disjoint, } \\
& \sum_{m=1}^{M} \sum_{k \in \mathcal{K}_{m}} P_{m, k} \leq P_{\text {total }}, \\
& \operatorname{BER}_{m} \leq P e_{m} \forall m \in \mathcal{M},
\end{aligned}
$$

where $\eta_{m}$ (bits/time slot) is the average long term throughput for user $m$ defined as,

$$
\eta_{m}=\lim _{t->\infty} \frac{1}{t} \sum_{n=1}^{t} \min \left(R_{m}(n), Q_{m}(n)\right) \forall m \in \mathcal{M} .
$$

$\mathcal{K}$ is the subcarrier index set $\{1,2, \ldots, K\}, \mathcal{K}_{m}$ is the set of subcarriers assigned to user $m, \mathcal{M}$ is the user index set $\{1,2, \ldots, M\}, P_{m, k}$ is the power allocated to the user $m$ on subcarrier $k, P_{\text {total }}$ is the total power constraint, $\mathrm{BER}_{m}$ is the bit error rate for user $m$ and $P e_{m}$ is the bit error rate constraint for user $m$. A delay constraint is imposed by the finite buffer size for each queue. For the sake of simplicity, a subcarrier is allocated to only one user at any instant [4].

\section{JSPA FOR CAQA SCHEDULING}

The MLWDF rule is proposed in [1], [12] for use in time slot allocation in CDMA-HDR systems. It has been proved in [1] that the MLWDF rule is throughput optimal in terms of slot allocation. In [1], the base station scheduler allocates the entire transmission bandwidth and power to a single user i.e., all spreading codes and all the available power are assigned to a single user during a time slot. This algorithm can be applied to OFDM systems under the constraint that only one user is allocated all the subcarriers and power in each time slot.

Let $\gamma_{m, k}(n)$ be the channel gain to noise power ratio for user $m$ on subcarrier $k, \mathbf{X}_{m}(n)$ be a set whose elements are the channel gain to noise power ratios of all subcarriers for user $m$, i.e., $\mathbf{X}_{m}(n)=\left\{\gamma_{m, 1}(n), \gamma_{m, 2}(n), \cdots, \gamma_{m, K}(n)\right\}$. $A_{m}(n)=f\left(\mathbf{X}_{m}(n), P_{\text {total }}, P e_{m}\right)$ is the achievable rate for user $m$ during time slot $n$ if all bandwidth and power were to be allocated to this user. The function $f($.$) denotes the$ algorithm in [13] and maximizes the user's achievable rate based on: (i) the channel gain to noise power ratio on the allocated subcarriers, (ii) the total available power for the user, and (iii) the BER constraint for the user. Furthermore, the rate on each subcarrier is constrained to take on values only from a discrete rate set using integer bit constellations such as MQAM. $\bar{A}_{m}=\lim _{t->\infty} \frac{1}{t} \sum_{n=1}^{t} A_{m}(n) \forall m \in \mathcal{M}$ is the mean achievable rate for user $m$ during a time slot assuming full bandwidth and power allocation.

The MLWDF rule chooses the user $i$ with the largest MLWDF parameter from amongst all users during a time slot i.e.,

$$
i=\arg \max _{m} \frac{A_{m}(n) W_{m}(n)}{\bar{A}_{m}} \forall m \in \mathcal{M}
$$


Therefore, $R_{i}(n)=A_{i}(n)$ and $R_{j}(n)=0 \forall j \neq i$. However, scheduling a single user for transmission during a time slot can lead to under utilization of the available bandwidth and power [4], [11]. If user $i$ is scheduled during time slot $n$, it is quite possible that $R_{i}(n)>Q_{i}(n)$, leading to a wastage in resources. If more than one user is allowed to transmit during a time slot, this wastage of resources can be minimized. In [2], this is accomplished by applying the MLWDF rule to allocate one subcarrier at a time during a time slot. Uniform power allocation is assumed in order to calculate the achievable rates. In [3], a similar subcarrier allocation algorithm using mean waiting time instead of HOL delay is proposed. Simulation results in [2] show that using instantaneous HOL delay is better than using mean waiting time [3].

In [2], [3], the HOL delay or the average waiting time are updated once a slot. However, these updated delays could be estimated after each subcarrier is allocated. In the CAQA scheduling scheme with fixed power allocation (FPA) considered in this paper, a user's queue is estimated immediately after a subcarrier is allocated to a user. This estimated queue information is used in the allocation of the remaining subcarriers. Therefore, the CAQA scheduling scheme with FPA used in this paper is superior to the scheduling schemes outlined in [2], [3].

\section{A. Proposed Algorithm}

In the proposed JSPA approach, the power allocation of a user is optimized every time a subcarrier is allocated to that user. This updated power allocation is used for the allocation of the remaining subcarriers and can, therefore, provide significant performance gain. CAQA scheduling with JSPA during time slot $n$ is as given below. $N_{m}(n)$ is the number of subcarriers allocated to user $m$ during time slot $n, A_{m, k}(n)=f\left(\gamma_{m, k}(n), \frac{P_{\text {total }}}{K}, P e_{m}\right)$ is the achievable rate for user $m$ on subcarrier $k$ with available power $\frac{P_{\text {total }}}{K}$, $\bar{A}_{m, k}=\lim _{t->\infty} \frac{1}{t} \sum_{n=1}^{t} A_{m, k}(n)$ is the mean achievable rate for user $m$ on subcarrier $k$ with available power $\frac{P_{\text {total }}}{K}$ during a time slot.

1) Initialize: $\forall m \in \mathcal{M}, \mathbf{X}_{m}(n)=\{\}, N_{m}(n)=0$, $R_{m}(n)=0, W_{m}^{\prime}(n)=W_{m}(n) \forall m \in \mathcal{M}$

2) Allocate one subcarrier at a time: For each subcarrier $k=1$ to $K$

a) Allocate the subcarrier to a user: select user $j$ such that,

$j=\arg \max _{m}\left[\frac{A_{m, k}(n) W_{m}^{\prime}(n)}{A_{m, k}}\right] \forall m \in \mathcal{M}$

b) $\mathbf{X}_{j}(n)=\mathbf{X}_{j}(n) \cup \gamma_{j, k}(n), N_{j}(n)=N_{j}(n)+1$

c) Optimize power allocation for the user: $R_{j}(n)=$ $f\left(\mathbf{X}_{j}(n), \frac{P_{t o t a l}}{K} N_{j}(n), P e_{j}\right)$

d) Estimate $W_{j}^{\prime}(n)$ as follows:

$$
\begin{aligned}
& W_{j}^{\prime}(n)=\arg \max _{1 \leq W \leq D}\left\{R_{j}(n)-\sum_{d=W-1}^{D-1} q_{j, d}(n)<0\right\} \\
& \text { if } R_{j}(n)<Q_{j}(n) \text {, and } W_{j}^{\prime}(n)=0 \text { if } R_{j}(n) \geq Q_{j}(n) .
\end{aligned}
$$

In step 2(a), note that the estimate of the HOL packet delay of each of the users is used for the purpose of allocating a subcarrier to a user. JSPA is achieved in step 2(c) of the algorithm by optimizing power allocation across all subcarriers currently allocated to the user during this time slot. This maximizes the user's rate thereby making best possible use of the allocated bandwidth and power. Step 2(d) is used to determine the new HOL delay after the rate allocated in step 2(c) is used. If during step 2(c), it so happens that $R_{j}(n) \geq Q_{j}(n)$, then step 2(d) ensures that user $j$ will no longer be in contention for subcarrier and power allocation. Therefore, a user will not be allocated more bandwidth and power than is needed to transmit all bits in the queue.

\section{B. Complexity of JSPA}

The complexity of subcarrier allocation is identical for both CAQA scheduling with FPA and CAQA scheduling with JSPA. However, unlike the FPA algorithm, the proposed JSPA requires power and bit allocation to be optimized after each subcarrier is allocated. The power and bit allocation is performed in step 2(c) using the algorithm in [13], which uses the bisection method with rate-SNR (Symbol energy to Noise power Ratio) look-up tables to compute the optimal power and bit allocation. The complexity of the algorithm in [13] is proportional to the product of the number of subcarriers over which the optimization needs to be performed and the number of iterations required for convergence. The additional complexity of the JSPA algorithm is not significant compared to the complexity of the CAQA algorithm with FPA because: (a) the number of subcarriers over which the optimization has to be performed in lower for the intermediate optimization stages, (b) when each additional subcarrier is allocated to a user, fewer iterations are required for convergence [13] since a good initialization point is already available from the previous rate maximization result for the user, and (c) subcarrier allocation, whose complexity is similar for both FPA and JSPA algorithms, is still the more complex part of the overall algorithm.

\section{Band-Wise Allocation of Subcarriers}

CAQA scheduling with JSPA can be extended to bandwise allocation of subcarriers [14] to help reduce the signaling overhead. On the uplink, the overhead includes feedback of the CSI of subcarriers for each user in the system. On the downlink, the updated subcarrier allocation and modulation information needs to be signalled to each user every time the allocation changes. A user's channel on adjacent subcarriers is correlated: the correlation is dependent on the user's multipath delay spread. Therefore, it is possible to reduce signaling overhead for both uplink and downlink by grouping adjacent subcarriers into sub-bands and limiting the sub-band CSI feedback to the CSI of the worst subcarrier in a sub-band (in order to meet the BER constraint). All subcarriers in a subband will be allocated equal power and the same modulation scheme.

\section{Simulation Results}

Results are shown for a $K=128$ subcarrier multiuser OFDM system with $M=12$ users and bandwidth $B=1$ MHz. Each user is assumed to have a 6-tap sample-spaced multipath channel with independent Rayleigh fading taps. The 


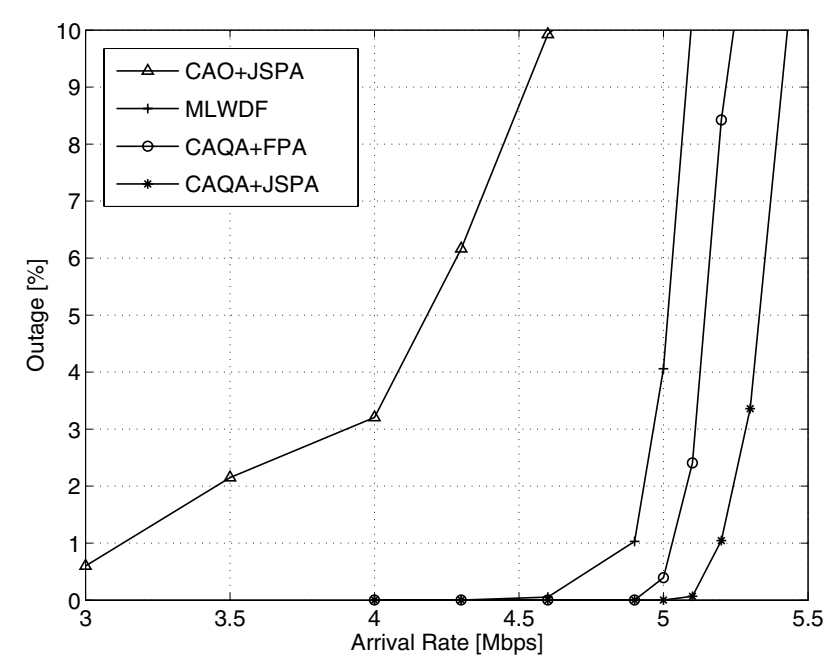

Fig. 1. Outage vs arrival rate: Case $1, P_{\text {total }}=8 \mathrm{dBW}$.

tap energies are assumed to decay exponentially as in [8], i.e., $E\left[\left|h_{l, m}\right|^{2}\right]=g_{m} e^{-(l-1)}$ for $l=1,2, . ., 6, m \in \mathcal{M}$ where $h_{l, m}$ is the time domain tap $l$ for user $m$ and $g_{m}$ is the tap energy of the first tap for user $m$. The BER constraint is $P e_{m}=10^{-5} \forall m \in \mathcal{M}$. Instantaneous SNR for user $m$ on subcarrier $k$ is defined as $\mathrm{SNR}=P_{m, k}(n) \gamma_{m, k}(n)$. The $\mathrm{SNR}$ thresholds for a BER of $10^{-5}$ for BPSK, QPSK, 8-QAM, 16QAM, 32-QAM and 64-QAM are $9.25 \mathrm{~dB}, 12.51 \mathrm{~dB}, 16.12 \mathrm{~dB}$, $19.32 \mathrm{~dB}, 22.47 \mathrm{~dB}$ and $26.03 \mathrm{~dB}$ respectively.

The source arrival for each user is an independent Bernoulli random process with the incoming packet size fixed at 128 bytes. The queue for each user is a FIFO buffer designed to hold packets for a maximum of 100 time slots. If a packet is not scheduled for transmission within 100 time slots, the packet is dropped from the queue. In simulations, we have assumed that all users' queues are stable if the bit outage probability is less than 0.5 percent for each user i.e., $\eta_{m} \geq$ $0.995 \lambda_{m} \quad \forall m \in \mathcal{M}$. Each simulation is performed for at least 10000 time slots. We compare CAQA scheduling with JSPA (CAQA+JSPA) with: (1) CAO scheduling with JSPA (CAO+JSPA) - Equivalent to [8] with an integer bit constraint, (2) MLWDF rule [1] - An OFDM/TDMA scheme, and (3) CAQA scheduling with FPA (CAQA+FPA) - Improved version of [2] (See Section III).

\section{A. Case 1 Homogeneous Rate Users With Different Channel Conditions}

In this case, the average rate of arrival of bits is equal for all users i.e., $\lambda_{m}=\lambda_{j} \forall m, j \in \mathcal{M}$. However, the average channel conditions for each user in the system is different i.e., $g_{m}=(1-m) \mathrm{dB}$ for $m=1,2, \ldots, M$. Noise Power Spectral Density (PSD) is set at $-100 \mathrm{dBW} / \mathrm{Hz}$.

Fig. 1 shows the maximum outage among all users for different overall arrival rates for $P_{\text {total }}=8 \mathrm{dBW}$. Clearly, the proposed algorithm is able to maintain the queues stable for a larger traffic load. The average long-term throughput is the same as the arrival rate as long as the outage is zero. Outage occurs once the arrival rate increases beyond a maximum arrival rate. Using a stability criterion of less than $0.5 \%$ outage

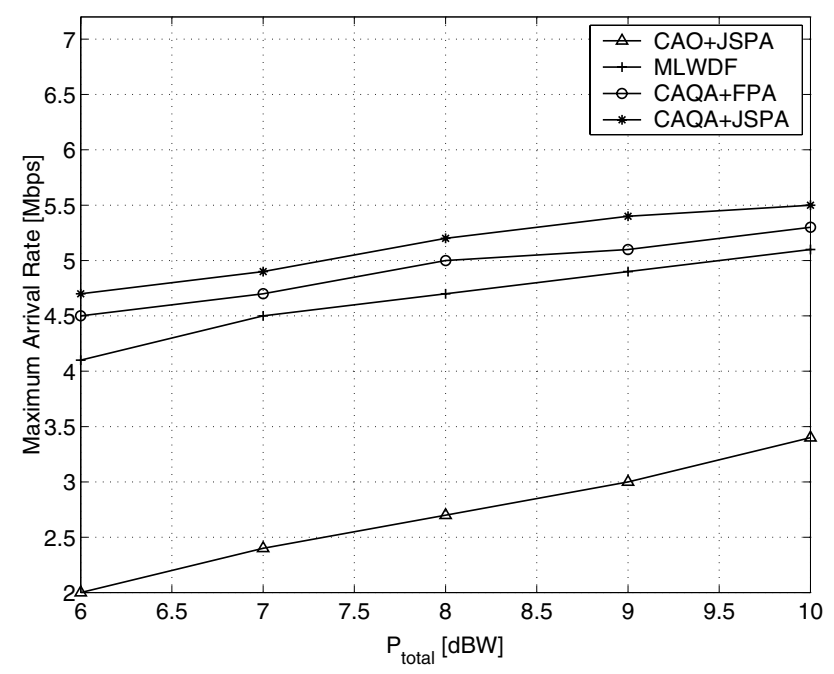

Fig. 2. Maximum arrival rate vs power: Case 1.

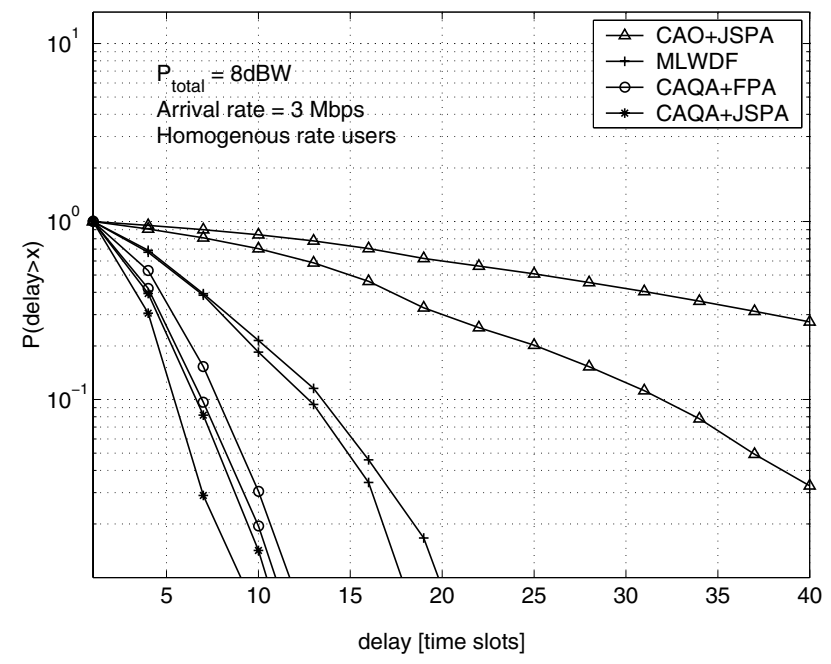

Fig. 3. Delay distribution tails for the best and worst users for each algorithm: Case 1.

for each user, this maximum arrival rate can be determined for a given $P_{\text {total }}$ from Fig. 1. Fig. 2 shows the largest arrival rate with stable queues for different values of $P_{\text {total }}$ for all the scheduling schemes. CAQA scheduling with JSPA achieves a gain of about $2 \mathrm{~dB}$ and $1 \mathrm{~dB}$ over MLWDF and CAQA scheduling with FPA respectively. Fig. 3 shows the delay distribution tails plotted as a function of the number of time slots for the best and the worst users when the total available power $P_{\text {total }}$ is $8 \mathrm{dBW}$ and overall arrival rate is $3 \mathrm{Mbps}$. A delay violation probability of $10^{-1}$ corresponds to a 10 time slot delay for CAQA scheduling and around a 35 time slot delay for CAO scheduling. From Fig. 3, it is evident that CAQA scheduling with JSPA provides a lower delay violation probability compared to CAQA scheduling with FPA and MLWDF.

Fig. 4 shows the largest arrival rate with stable queues for band-wise allocation of subcarriers with different sub-band sizes for CAQA scheduling with JSPA. This is plotted for different values of the rms multipath delay spread. $L$ is the number of subcarriers per sub-band and the total available power $P_{\text {total }}=6 \mathrm{dBW}$. When the rms multipath delay spread 


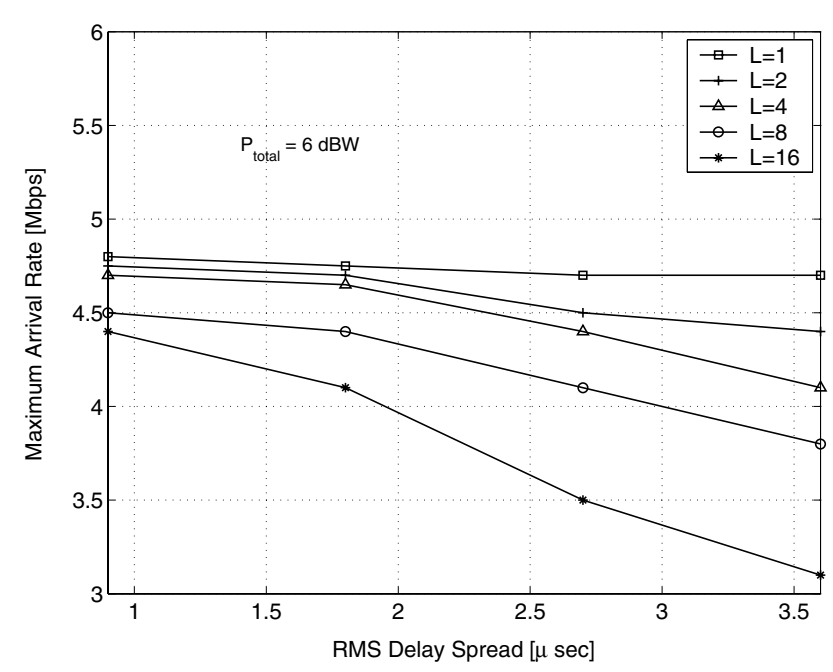

Fig. 4. Arrival vs. RMS delay spread: Band-wise allocation.

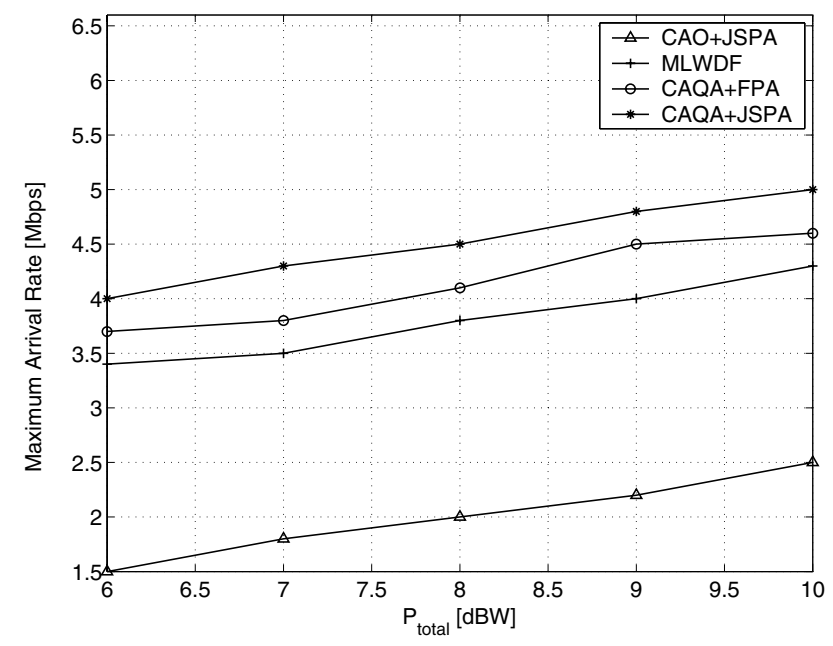

Fig. 5. Maximum arrival rate vs power: Case 2.

is set at $0.9 \mu \mathrm{sec}$, a signaling overhead reduction by a factor $L=2$ or $L=4$ can be achieved with hardly any performance degradation. This is because, the coherent bandwidth is large and the sub-band CSI provides a fairly accurate estimate of the CSI of subcarriers in the sub-band. However, with increasing delay spread, the CSI of sub-bands provide increasingly pessimistic estimates of the CSI of subcarriers in a particular subband. As a result, power and bandwidth allocation efficiency falls with increasing sub-band size. When the rms delay spread is $3.6 \mu \mathrm{sec}$, even a sub-band size as small as $L=4$ results in a 10 percent degradation relative to $L=1$.

\section{B. Case 2 Heterogeneous Rate Users With Similar Channel Conditions}

In this case, the average channels conditions are the same for each user in the system $g_{m}=g_{j} \forall m, j \in \mathcal{M}$. However, the average rate of arrival of bits is different for each of the users. $\lambda_{m}=\lambda_{1}(1+0.25(m-1))$ for $m=1$ to 6 , and $\lambda_{m}=$ $\lambda_{1}(1+0.25(m))$ for $m=7$ to 12 .

In Fig. 5, the total available power $P_{\text {total }}$ is varied and the largest arrival rate with stable queues is plotted for the

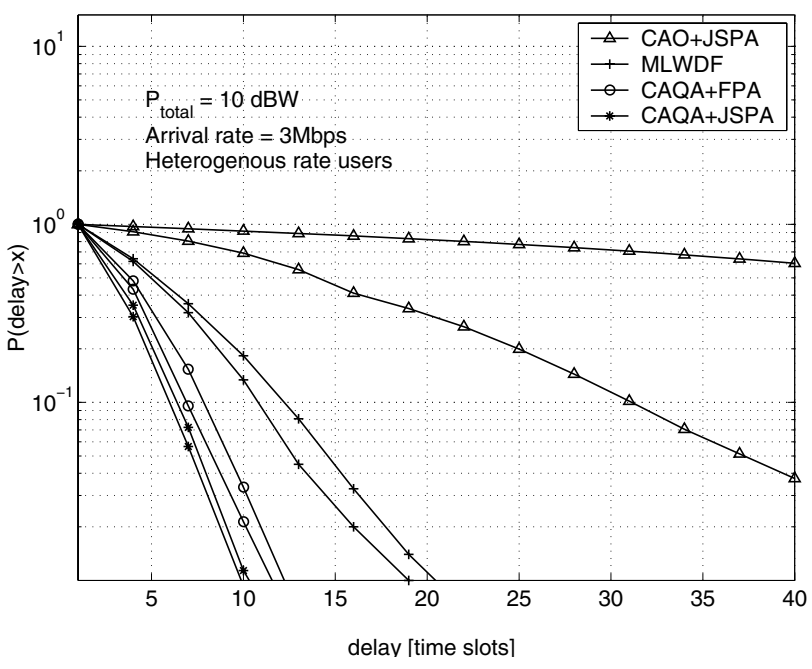

Fig. 6. Delay distribution tails for the best and worst users for each algorithm: Case 2.

scheduling schemes. Noise PSD is set at $-90 \mathrm{dBW} / \mathrm{Hz}$. CAQA scheduling with JSPA achieves an approximate gain of $2 \mathrm{~dB}$ and $1 \mathrm{~dB}$ over MLWDF and CAQA scheduling with FPA respectively. Fig. 6 shows the delay distribution tails plotted as a function of the number of time slots for the best and the worst users when the total available power $P_{\text {total }}$ is 10 $\mathrm{dBW}$ and overall arrival rate is $3 \mathrm{Mbps}$. As in the case with homogeneous rate users, it is evident that CAQA scheduling with JSPA provides for a lower delay violation probability compared to CAQA scheduling with FPA and MLWDF.

\section{CONCLUSIONS}

In this paper, a sub-optimal joint subcarrier and power allocation algorithm for Channel-Aware Queue-Aware scheduling on a multiuser OFDM downlink is proposed. Our approach is to optimize a user's power allocation immediately after each subcarrier is allocated to the user. Through simulations, performance gains of 1-2dB over existing methods have been shown. The proposed approach was also extended to bandwise allocation of subcarriers in order to reduce the signaling overhead while still achieving good system performance.

\section{REFERENCES}

[1] M. Andrews, K. Kumaran, K. Ramanan, A. Stolyar, R. Vijayakumar, and P. Whiting, "CDMA data QoS scheduling on the forward link with variable channel conditions," Bell Labs Technical Memorandum, 2000.

[2] P. Parag, S. Bhashyam, and R. Aravind, "A subcarrier allocation algorithm for OFDMA using buffer and channel state information," in Proc. 62nd IEEE Veh. Technol. Conf., Sep. 2005, vol. 1, pp. 622-625.

[3] G. Song, Y. Li, L. J. Cimini, and H. Zheng, "Joint channel-aware and queue-aware data scheduling in multiple shared wireless channels," in Proc. IEEE WCNC, Mar. 2004, vol. 3, pp. 1939-1944.

[4] C. Y. Wong, R. S. Cheng, K. B. Letaief, and R. D. Murch, "Multiuser OFDM with adaptive subcarrier, bit, and power allocation," IEEE J. Select. Areas Commun., vol. 17, no. 10, pp. 1747-1758, Oct. 1999.

[5] J. Jang and K. B. Lee, "Transmit power adaptation for multiuser OFDM systems," IEEE J. Select. Areas Commun., vol. 21, no. 2, pp. 171-178, Feb. 2003.

[6] W. Rhee and J. M. Cioffi, "Increase in capacity of multiuser OFDM system using dynamic subchannel allocation," in Proc. 51st IEEE Veh. Technol. Conf., May 2000, vol. 2, pp. 1085-1089.

[7] Z. Shen, J. G. Andrews, and B. L. Evans, "Adaptive resource allocation in multiuser OFDM systems with proportional fairness," IEEE Trans. Wireless Commun., vol. 4, no. 6, pp. 2726-2737, Nov. 2005. 
[8] C. Mohanram and S. Bhashyam, "A sub-optimal joint subcarrier and power allocation algorithm for multiuser OFDM," IEEE Commun. Lett., vol. 8, pp. 685-687, Aug. 2005.

[9] G. Song and Y. Li, "Cross-layer optimization for OFDM wireless networks-Part I: Theoretical framework," IEEE Trans. Wireless Commun., vol. 4, no. 2, pp. 614-624, Mar. 2005.

[10] G. Song and Y. Li, "Cross-layer optimization for OFDM wireless networks-Part II: Algorithm development," IEEE Trans. Wireless Commun., vol. 4, no. 2, pp. 625-634, Mar. 2005.

[11] K. Kumaran and H. Viswanathan, "Joint power and bandwidth allocation in downlink transmission," IEEE Trans. Wireless Commun., vol. 4, no. 3, pp. 1008-1016, May 2005.
[12] M. Andrews, K. Kumaran, K. Ramanan, A. Stolyar, P. Whiting, and R. Vijayakumar, "Providing quality of service over a shared wireless link," IEEE Commun. Mag., vol. 39, no. 2, pp. 150-154, Feb. 2001.

[13] B. S. Krongold, K. Ramachandran, and D. L. Jones, "Computationally efficient optimal power allocation algorithms for multicarrier communication systems," IEEE Trans. Commun., vol. 48, no. 1, pp. 23-27, Jan. 2000 .

[14] J. Oh and J. M. Cioffi, "Sub-band rate and power control for wireless OFDM," in Proc. 60th IEEE Veh. Technol. Conf., Sep. 2004, vol. 3, pp. 2011-2014. 